\title{
Application of Triboelectric Nanogenerator in Smart Home and Clothing
}

\author{
Kaiqin Zhao and Zhiyuan Zhu* \\ School of Electronic Information Engineering, Southwest University, Chongqing, China
}

Keywords: triboelectronic nanogenerator, energy, self-power, smart home, clothing

\section{INTRODUCTION}

The most important energy conversion in human history is combustion, such as the use of coal, oil, etc., which are usually inefficient and causes serious environmental problems. Thus, realizing efficient energy conversion and storage always attract the interest of scientific research. Despite the rapid development of modern batteries such as fuel cells and lithium-ion batteries, due to the

OPEN ACCESS

Edited by:

Xiaosheng Zhang

University of Electronic Science and

Technology of China, China

Reviewed by:

Zhihua Pu,

Tianjin University, China

Hongye Liu,

University of Shanghai for Science and

Technology, China

*Correspondence:

Zhiyuan Zhu

zyuanzhu@swu.edu.cn

Specialty section:

This article was submitted to Micro- and Nanoelectromechanical

Systems,

a section of the journa

Frontiers in Mechanical Engineering

Received: 27 June 2020

Accepted: 28 August 2020

Published: 06 October 2020

Citation:

Zhao K and Zhu Z (2020) Application

of Triboelectric Nanogenerator in

Smart Home and Clothing.

Front. Mech. Eng. 6:576896. doi: 10.3389/fmech.2020.576896 huge distribution of battery usage and short life span, great efforts are still required for battery monitoring, replacement, and recycling. In addition, inevitable phenomena such as mechanical vibration or parts friction cause energy dissipation. Therefore, developing clean and efficient energy or improving energy utilization has become a hot issue which are related to world peace and economic development. Collecting and using energy lost by friction is an environmentally friendly and effective way to improve energy efficiency. Since Prof. Wang Zhonglin's nano research team invented the triboelectric nanogenerator (TENG) in 2012, it has made many breakthroughs in the basic mechanism and self-driving system of TENG. TENG has many advantages in micro-nano energy harvesting and can be widely used in sensors, portable devices, etc.

\section{BASIC PRINCIPLE}

The principle of TENGs is based on the coupling of triboelectric charging and electrostatic induction (Li et al., 2017). Generally, when two materials contact each other, chemical bonds are formed at some parts of the interface, and charges are transferred between the interfaces to balance the electrochemical potential, thereby generating triboelectric charges. Driven by external forces, the frictionally charged interfaces move with each other, causing the potential difference in TENGs to change periodically. Under short circuit conditions, an alternating current flows through the load to achieve an electrostatic balance between the two electrodes.

It has four basic modes (Niu et al., 2013; Yang et al., 2013): (a) vertical contact-separation mode; (b) lateral sliding mode; (c) single electrode mode; (d) self-supporting triboelectric layered mode.

Since 2012, four different modes of TENGs and self-powered electrochemical systems based on it have been developed. The research progress mainly includes the following aspects: First, in order to use different mechanical sources and meet different practical applications, a variety of structures have been designed, including spring-supported, arched, saw tooth, grating structure, and multilayer Structure, etc. Second, different self-powered systems have been designed to collect various energy sources, including human motion, engine rotation, wind energy, and water energy. Third, a variety of applications based on TENGs have been reported, especially self-powered sensors, which can be divided into two categories: physical sensors and chemical sensors. 


\section{DISCUSSION}

The working principle of the triboelectric nanogenerator determines that its development trend is sustainable, flexible, efficient, low cost, and environmental friendly design. It will be used to build ultrasensitive sensors, microelectromechanical devices, wearable electronics, self-powered systems such as environmental protection and new energy technologies provide a viable solution to the global energy crisis.

\section{Application of TENG in Smart Home}

For most smart homes, it is externally powered. After the appearance of the friction nano-generator, it can be equipped with sensors that can respond to changes in the environment without a power supply to control the smart home.

\section{Example 1}

The triboelectric nanogenerator can be used as a switch sensor for smart home lighting fixtures. At present, the light switch in the smart home can be divided into voice control switch and light control switch. However, the lamps controlled by these two switches in the smart home need to be automatically turned off for a period of time when turned on, which results in the consumption and waste of electrical energy. Suppose TENG is made into a wooden floor as a switch sensor, the mechanical energy of people walking can be harvested and converted into electrical energy, it does not require external power, so there is no need for a light switch. It can effectively avoid the problem that the lighting time is longer than the actual time, and effectively save energy.

\section{Example 2}

It is possible to design a triboelectric nanogenerator integrated on the glasses frame, which detects tiny blinking motions and converts it into a voltage signal, which is applied to two real-time mechanical man-machine interfaces-smart home control systems and Wireless hands-free typing system (Pu et al., 2017).

\section{Example 3}

Under friction, the magnitude of the voltage generated by the nanogenerator and the magnitude and frequency of the externally applied force rate-related features, triboelectric nanogenerator are used in home floors as a sensor for pressure detection.

\section{Example 4}

When TENG is installed under the carpet at the door or on the door handle, it can play when someone breaks in the role of security warning. Moreover, the TENG can be made into a matrix installed under the floor, according to the location of the electrical signal to locate the outsider. The position of the outsider on the floor can also pass the information through Bluetooth, WiFi, etc. Wireless transmission mode, transmitted to the monitoring camera control system to enable the camera. The tracking and positioning of the case is helpful to the investigation of the case and the collection of evidence.

\section{Application of TENG in Clothing}

Fabric-based smart wearable devices, as a combination of Internet technology and modern micro-intelligence manufacturing in the field of textiles and garments, put forward requirements for electric power supply systems that are smaller in size, longer in durability, flexible and easy to integrate, low in overall cost, clean and environmentally friendly. TENG can be used in this aspect very well.

\section{Example 5}

The acceleration sensor based on the triboelectric nanogenerator can be placed on the sole of the shoe to effectively record the frequency and acceleration of people running.

\section{Example 6}

A special material is used to form TENG on the clothes, and then an electrical signal is generated through the friction of the body's activities to provide energy for the heating system of the clothes. The TENG on clothes can also be used as an energy collector to absorb static electricity on clothes and reduce the phenomenon of static electricity on clothes.

\section{Example 7}

The TENG can also be integrated in smart gloves to achieve gesture sensing. When the finger is bent or straightened, the contact area of the skin and the TENG will change, and the realtime voltage will change accordingly, so as to realize the induction of different gestures.

\section{Example 8}

The TENG structure is attached to the insole and generates an electrical signal when walking. On the one hand, it acts as an energy conversion device to collect energy, on the other hand, it can directly sterilize the insole.

\section{MINIATURIZATION PROSPECT}

At the current stage, the current output power of the TENG system is generally small, and the friction charge density needs to be further improved. The randomness of the friction activity generates the friction force, contact angle, etc. In each friction cycle have undeterminable differences, resulting in unstable output power of the TENG. The electrical resistance in the electrical circuit of the triboelectric system is too large to provide direct and effective power supply to the electronic components, so this system is generally used in the field of real-time sensing of small power at the present stage. Because the current power converted from low-frequency energy is too small to be used for charging, there is still a bottleneck in the promotion of the development of the complete energy supply supporting system based on the TENG. In practical applications, most of the electrical signals output by the TENG are AC electrical signals, which require an external rectifier bridge or capacitor, which brings adverse influence to miniaturization.

In response to these bottlenecks, it is possible to adapt to the randomness of frictional motion by combining multiple 
TENG models and modifying the shape of the friction surface; selecting the appropriate friction layer and dielectric material can effectively reduce the electrical circuit resistance; by increasing the charge density on the friction surface and speeding up Increase the output current power by friction cycle, increasing friction area, etc.; based on the current system current realtime output characteristics, design the energy storage and deployment system to adapt to the energy generation system, and finally design a friction nanometer with excellent electrical output performance and fabric performance generator. In terms

\section{REFERENCES}

Li, M., Porter, A. L., and Wang, Z. L. (2017). Evolutionary trend analysis of nanogenerator research based on a novel perspective of phased bibliographic coupling. Nano Energy 34, 93-102. doi: 10.1016/j.nanoen.2017.02.020

Niu, S., Wang, S., Lin, L., Liu, Y., Zhou, Y. S., Hu, Y., et al. (2013). Theoretical study of con? tact-mode triboelectric nanogenerators as an effective power source. Energy Environ. Sci. 12, 3576-3583. doi: 10.1039/c3ee42571a

Pu, X., Guo, H., Chen, J., Wang, X., Xi, Y., Hu, C., et al. (2017). Eye motion triggered selfpowered mechnosensational communication system using triboelectric nanogenerator. Sci. Adv. 7:1700694. doi: 10.1126/sciadv.1700694

Yang, Y., Zhang, H., Chen, J., Jing, Q., Zhou, Y. S., Wen, X., et al. (2013). Single-electrode based sliding triboelectric nanogenerator for of energy conversion, it is possible to increase the research on new structures such as constant-current TENG that do not require external capacitors, and promote their intelligence and miniaturization.

\section{AUTHOR CONTRIBUTIONS}

All authors listed have made a substantial, direct and intellectual contribution to the work, and approved it for publication.

self-powered displacement vector sensor system. Acs Nano 8, 7342-7351. doi: $10.1021 / \mathrm{nn} 403021 \mathrm{~m}$

Conflict of Interest: The authors declare that the research was conducted in the absence of any commercial or financial relationships that could be construed as a potential conflict of interest.

Copyright (c) 2020 Zhao and Zhu. This is an open-access article distributed under the terms of the Creative Commons Attribution License (CC BY). The use, distribution or reproduction in other forums is permitted, provided the original author(s) and the copyright owner(s) are credited and that the original publication in this journal is cited, in accordance with accepted academic practice. No use, distribution or reproduction is permitted which does not comply with these terms. 